\title{
Nonlinear Properties of Silicon Optical Fibers
}

\author{
A. C. Peacock ${ }^{1}$, P. Mehta ${ }^{1}$, T. D. Day ${ }^{2}$, J. R. Sparks ${ }^{2}$, P. J. A. Sazio ${ }^{1}$, J. V. Badding ${ }^{2}$, \\ and N. Healy ${ }^{1}$ \\ 1. Optoelectronics Research Centre, University of Southampton, Southampton SO17 1BJ, United Kingdom \\ 2. Department of Chemistry and Materials Research Institute, Pennsylvania State University 16802 PA, USA \\ acp@orc.soton.ac.uk
}

\begin{abstract}
The nonlinear transmission properties of hydrogenated amorphous silicon core fibers are characterized for short pulse propagation. Simple pump-probe experiments are employed to demonstrate their use for all-optical control via phase and amplitude modulation.

(C) 2012 Optical Society of America

OCIS codes: $060.2270,060.2290,060.4370$.
\end{abstract}

\section{Introduction}

Silicon waveguides are an ideal platform for nonlinear optical signal processing due to their large Kerr nonlinearity and high core/silica-cladding index contrast which allows for tight mode confinement. As such, a number of all-optical devices have been demonstrated in rectilinear silicon waveguides on-chip including optical regeneration, modulation and switching. The incorporation of silicon into the optical fiber geometry provides an important step towards integrating semiconductor functionality with conventional fiber infrastructures [1]. In this paper we review our progress in characterizing the nonlinear transmission properties of the silicon core fibers that are fabricated using a high pressure chemical deposition technique [2]. Although typical core sizes for these fibers are of the order of a few micron, the high nonlinear index of the hydrogenated amorphous silicon core material has enabled the observation of substantial spectral broadening in spite of the presence of both linear and nonlinear losses. Using simple pump-probe experiments we have demonstrated the capability for all-optical control using both cross-phase modulation (XPM) and cross-absorption modulation (XAM) [3].

\section{Theory and Experiment}

Nonlinear pulse propagation in silicon fibers can be described by a modified form of the nonlinear Schrödinger equation (NLSE), together with a coupled equation for the free carrier density $N_{c}$ [2]:

$$
\begin{aligned}
\frac{\partial A}{\partial z} & =-\frac{i \beta_{2}}{2} \frac{\partial^{2} A}{\partial t^{2}}+i \gamma|A|^{2} A-\frac{1}{2}\left(\sigma_{f}+\alpha_{l}\right) A, \\
\frac{\partial N_{c}}{\partial t} & =\frac{\beta_{\mathrm{TPA}}}{2 h v_{0}} \frac{|A|^{4}}{A_{\mathrm{eff}}^{2}}-\frac{N_{c}}{\tau_{c}} .
\end{aligned}
$$

Here $A$ is the pulse envelope, $\alpha_{l}$ is the linear loss, $\beta_{2}$ is the group velocity dispersion, and the complex nonlinearity parameter is defined as: $\gamma=k_{0} n_{2} / A_{\text {eff }}+i \beta_{\text {TPA }} / 2 A_{\text {eff }}$ in terms of the Kerr $n_{2}$ and two-photon absorption (TPA) $\beta_{\text {TPA }}$

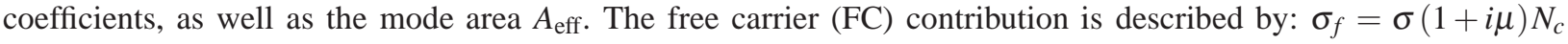
(absorption $\sigma$ and dispersion $\mu$ ) and $\tau_{c}$ is the carrier lifetime.

The fibers are fabricated using our deposition technique in which hydrogenated amorphous silicon (a-Si:H) is infiltrated into the pore of a silica capillary. Importantly, a-Si:H is becoming an increasingly popular material for nonlinear optical applications owing to its high $n_{2}$ and low optical losses. Fig. 1 shows the results following nonlinear characterization of a $5.7 \mu \mathrm{m}$ diameter core fiber with a linear loss determined via cut-back measurements of $\sim 3 \mathrm{~dB} / \mathrm{cm}$ at $1.54 \mu \mathrm{m}$. The TPA and FCA parameters are first determined via the nonlinear absorption measurements in Fig. 1(a), where we obtain $\beta_{\mathrm{TPA}} \sim 0.8 \mathrm{~cm} / \mathrm{GW}$ and $\sigma \sim 1 \times 10^{-16} \mathrm{~cm}^{2}$ from the fitted curve. Despite the relatively large linear and nonlinear losses, the spectral evolution displayed in Fig. 1(b) provides clear evidence of nonlinear broadening due to self-phase modulation (SPM) with $\sim 100 \mathrm{~nm}$ bandwidth. Using the predetermined linear and nonlinear loss parameters in Eqs. (1) and (2), we can fit these spectra to estimate a Kerr coefficient of $n_{2} \sim 1.5 \times 10^{-13} \mathrm{~cm}^{2} / \mathrm{W}$, as illustrated in Fig. 1(c). Although this value of $n_{2}$ is greater than twice that of crystalline silicon, similarly high values have also been reported for a-Si:H waveguides on-chip [4].

As a means of exploiting the large nonlinear parameters to demonstrate device functionality, a series of simple pump-probe experiments have been conducted. Fig. 2(a) shows the results of cross-phase modulation (XPM) induced by a strong pump pulse at $1.54 \mu \mathrm{m}$ acting on a weak probe at $1.59 \mu \mathrm{m}$, as a function of the pulse delay at the fiber 

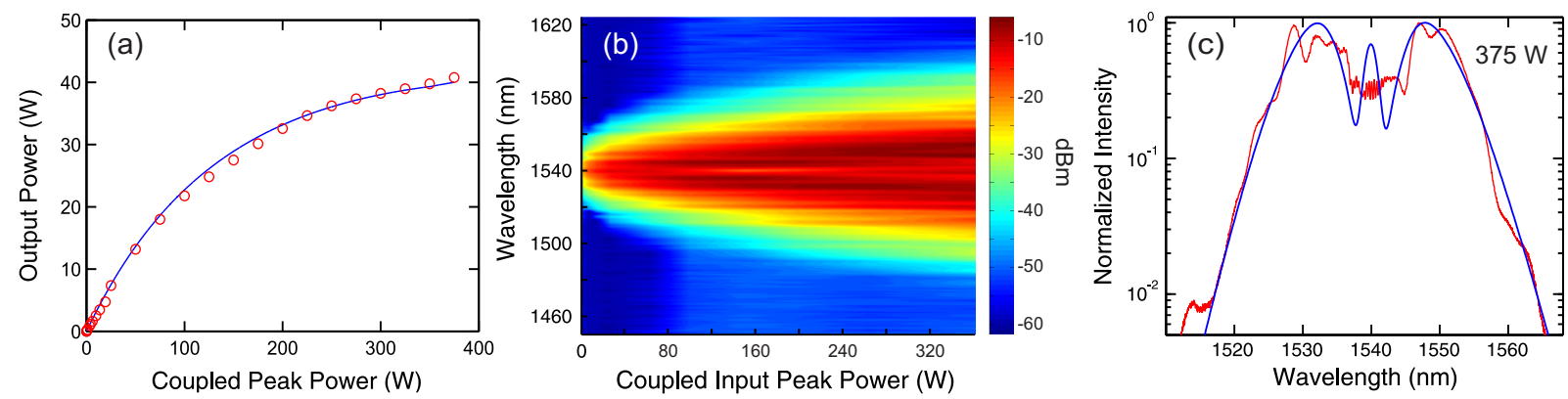

Fig. 1. Nonlinear characterization of a-Si:H fiber with a $5.7 \mu \mathrm{m}$ diameter core. (a) Nonlinear absorption and (b) SPM as functions of input power, (c) output spectrum with NLSE fit.

input. From this it is clear that when the probe propagates alone (large delays) that the power is sufficiently low to suppress SPM, whilst in the presence of the pump it experiences strong spectral modulation. By tailoring the spectral modulation to shift the central wavelength of the probe pulse, XPM can be used to add or drop channels in multiplexing schemes. Alternatively, the nonlinear losses can be exploited for all-optical modulation through XAM where the losses induced by a strong pump imprint a dark pulse on a weak signal. Fig. 2(b) shows the absorption on a probe at $1.57 \mu \mathrm{m}$ due to a $\sim 750 \mathrm{fs}$ pump pulse at $1.54 \mu \mathrm{m}$, as a function of delay. The results clearly show the ultra-fast response due to TPA, followed by a rapid recovery via carrier relaxation and then the slower recombination due to the free carriers, in agreement with a fit obtained from the coupled NLSEs [3]. Owing to the relatively large value of $\beta_{\text {TPA }}$ this modulation yields an extinction of $\sim 4 \mathrm{~dB}$, and this could be further improved by moving to shorter wavelengths. Unfortunately the long carrier lifetime determined from this measurement of $\tau_{c}=90 \mathrm{~ns}$ is a limiting factor for high speed device operation. However, by scaling the core size down towards the nanoscale dimensions used on-chip the free-carrier lifetime can be reduced and the nonlinear effects enhanced, paving the way for faster, lower power devices.
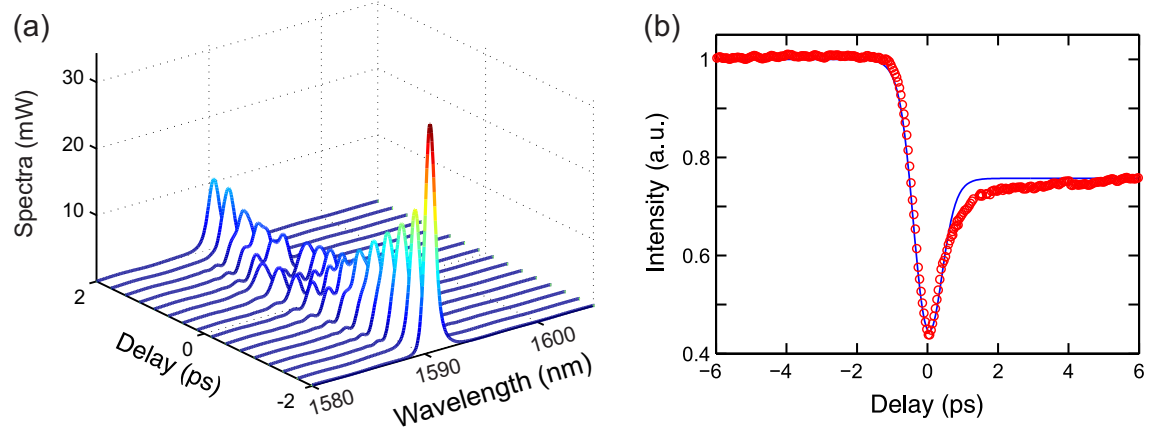

Fig. 2. (a) XPM: probe at $1.59 \mu \mathrm{m}$, pump at $1.54 \mu \mathrm{m}$. (b) XAM: probe at $1.57 \mu \mathrm{m}$, pump at $1.54 \mu \mathrm{m}$.

\section{Conclusion}

Investigations of the nonlinear properties of hydrogenated amorphous silicon core fibers have been presented. These fibers exhibit low optical losses and strong nonlinearities making them a suitable platform for the development of in-fiber nonlinear optical devices.

\section{References}

1. J. Ballato, T. Hawkins, P. Foy, R. Stolen, B. Kokuoz, M. Ellison, C. McMillen, J. Reppert, A. M. Rao, M. Daw, S. R. Sharma, R. Shori, O. Stafsudd, R. R. Rice, and D. R. Powers, "Silicon optical fiber," Opt. Express 16, 18675-18683 (2008).

2. P. Mehta, N. Healy, N. F. Baril, P. J. A. Sazio, J. V. Badding, and A. C. Peacock, "Nonlinear transmission properties of hydrogenated amorphous silicon core optical fibers," Opt. Express 16, 16826-16831 (2010).

3. P. Mehta, N. Healy, T. D. Day, J. R. Sparks, P. J. A. Sazio, J. V. Badding, and A. C. Peacock, "All-optical modulation using two-photon absorption in silicon core optical fibers," Opt. Express 19, 19078-19083 (2011).

4. B. Kuyken, H. Ji, S. Clemmen, S. K. Selvaraja, H. Hu, M. Pu, M. Galili, P. Jeppesen, G. Morthier, S. Massar, L.K. Oxenlwe, G. Roelkens, and R. Baets, "Nonlinear properties of and nonlinear processing in hydrogenated amorphous silicon waveguides,” Opt. Express. 19, B146-B153 (2011). 